\section{Diagnostische Instrumente zur \\ Erfassung von Störungen durch psychotrope Substanzen}

Zusammenfassung: Im Beitrag wird ein Überblick zu diagnostischen Instrumenten zur klassifikatorischen Diagnostik von Störungen durch psychotrope Substanzen gegeben, wie sie in den aktuellen Klassifikationssystemen ICD-10 und DSM-IV operationalisiert sind. Neben Checklisten haben vor allem strukturierte und standardisierte Interviews durch ihren hohen Grad an Zuverlässigkeit in den letzten Jahren zunehmend an Bedeutung gewonnen.

Schlüsselwörter: Diagnostik - Klassifikation - Instrumente - Checklisten - Strukturierte Interviews - Standardisierte Interviews

\begin{abstract}
Diagnostic-Instruments for the Assessment of Disorders Due to Psychoactives Substance Use: The current status on diagnostic instruments for classification of psychological and behavioral disorders due to psychoactive substance use are reviewed. Actual developments are oriented towards the operational concepts of ICD-10 and DSM-IV covering the most important substance abuse disorders. Especially checklists as well as structured and standardized interviews gained importance during the last years.
\end{abstract}

Key words: Diagnosis - Classification - Instruments Checklists - Structured Interviews - Standardized Interviews

\section{Einleitung}

Die Entwicklung und Einführung aktueller Klassifikationssysteme, wie DSM-III und ihre Nachfolger DSM-III-R und DSM-IV sowie ICD-10, sind eng verbunden mit der Entwicklung diagnostischer Instrumente zur Verbesserung der Reliabilität (vgl. [1]).

Umfassende psychiatrische Interviews wie das Composite International Diagnostic Interview (CIDI) oder die Schedules for Clinical Assessment in Neuropsychiatry (SCAN) wurden in diesem Kontext entwickelt und decken neben anderen psychischen Störungen vor allem auch Störungen bedingt durch psychotrope Substanzen ab.

Suchttherapie 2001; 2: 9-13

(c) Georg Thieme Verlag Stuttgart · New York ISSN 1439-9903
Rolf-Dieter Stieglitz ${ }^{1}$, Harald J. Freyberger ${ }^{2}$

${ }^{1}$ Psychiatrische Universitätspoliklinik Basel

${ }^{2}$ Klinik und Poliklinik für Psychiatrie und Psychotherapie der Ernst-Moritz-Arndt-Universität Greifswald im Klinikum Stralsund

Die große Zunahme im Gebrauch psychotroper Substanzen und damit verbundene Probleme im Gesundheitswesen unterstreichen die Notwendigkeit der Entwicklung von diagnostischen Instrumenten, die für unterschiedliche Zwecke in verschiedenen Settings Anwendung finden können. Üstun \& Wittchen ([2]; vgl. auch [3]) diskutierten die Bedeutung diagnostischer Instrumente, vor allem strukturierter Interviews, und betonen u.a. folgende wichtige Aspekte:

- Screening oder sog. Case-Finding, um möglichst frühzeitig potenzielle Fälle oder tatsächliche Fälle zu entdecken mit dem Ziel der Prävention und nachfolgender Intervention,

- reliable Erfassung von diagnostisch bedeutsamen Aspekten, wie Komorbidität, Muster von Abhängigkeiten und substanzbezogene Probleme im Hinblick auf eine systematische Behandlung, Rehabilitation und soziale Reintegration des Patienten,

- Interviews als Basis für die Evaluation von Symptommustern über verschiedene Substanzen im Hinblick auf den Verlauf und die natürliche Geschichte der Störung oder

- diagnostische Instrumente zur Evaluation von Interventionsprogrammen z. B. im Hinblick auf Kosteneffektivität.

\section{Instrumente zur klassifikatorischen Diagnostik}

Diagnosenstellungen im Rahmen etablierter Klassifikationssysteme wie ICD-10 oder DSM-IV können prinzipiell mittels eines klinischen Interviews oder durch die Anwendung von Instrumenten erfolgen. Die klinischen Interviews haben eine Reihe von Nachteilen, die vor allem dadurch bedingt sind, dass die bei der Beurteilung psychischer Phänomene in verschiedenen Studien identifizierten Fehlerquellen zum Tragen kommen:

- Informationsvarianz,

- Beobachtungs- oder Interpretationsvarianz.

Die Fehlerquelle Informationsvarianz ergibt sich vor allem dadurch, dass Diagnostiker unterschiedliche Fragen zur Erfassung und Bewertung der geforderten diagnostischen Kriterien stellen. Beobachtungsvarianz resultiert aus der unterschiedlichen Bewertung der erhobenen Informationen durch die Diagnostiker. Beide Varianzquellen sind als wichtige Faktoren im Hinblick auf eine mangelnde Übereinstimmung zwischen verschiedenen Urteilern zu werten, so dass allein die Anwendung von operationalisierten Diagnosekriterien nicht gewährleistet, dass eine hohe Übereinstimmung zwischen unterschiedlichen Beurteilern stattfindet. Als ein erster Schritt, den Beurteilungsprozess zu verbessern, können die sog. Checklisten angesehen werden. Diese zeichnen sich dadurch aus, dass die für die Diagnosenstellung relevanten 
Kriterien in einer didaktischen guten, meist übersichtlichen Form aufgelistet sind und deren Verknüpfung über einen Algorithmus erfolgt. Verschiedene Studien (vgl. z. B. [12,13]) haben zeigen können, dass derartige Checklisten zwar auch bei der Anwendung durch erfahrene Beurteiler zu einer hohen Reliabilität führen, jedoch das allgemeine Problem der Informations- und Beobachtungsvarianz dadurch nicht gelöst werden kann. Dementsprechend gehen weiterführende Ansätze dahin, beide Fehlerquellen stärker einzuschränken. Als erster Schritt in diese Richtung können die so genannten strukturierten Interviews angesehen werden, bei denen der Ablauf der Erhebung sowie die Vorgabe der Frage formalisiert sind. Auch hier bleibt jedoch die Frage der Beobachtungsvarianz bestehen, da in der Regel keine expliziten Kriterien zur Bewertung der von einem Patienten gegebenen Antworten vorliegen. Dies wird erst durch die sog. standardisierten Interviews erreicht, bei denen der gesamte Prozess der Informationserhebung klar festgelegt ist, d.h., welche Fragen in welcher Reihenfolge vorgegeben werden müssen, und zugleich Kodierungsregeln bestehen, wie Antworten von Seiten eines Patienten zu bewerten sind (vgl. hierzu auch [4]). Mittels dieser Vorgehensweise lässt sich eine hohe Reliabilität erreichen, was durch verschiedene Studien belegt werden konnte. Eine besondere Form der standardisierten Interviews stellen die so genannten computerisierten Versionen dar, bei denen zusätzlich der gesamte diagnostische Prozess computergeleitet gestützt wird. Nachfolgend sollen diejenigen Verfahren kurz skizziert werden, die im Kontext der Klassifikation von Störungen durch psychotrope Substanzen gegenwärtig in deutschsprachigen Versionen verfügbar sind.

Der Anwendung der zumeist auch zeitaufwändigen Instrumente vorgeschaltet werden können sog. Screeningverfahren, auf die daher zunächst kurz eingegangen werden soll.

\section{Screeningverfahren}

Screeningverfahren, wie sie oft auch in epidemiologischen Studien eingesetzt werden, haben die Funktion, mögliche „Risikopersonen“ zu identifizieren, um sie dann unter Umständen differenzierter nachzuuntersuchen. Da ein Screening eine Filterfunktion hat und möglichst ökonomisch erfolgen muss, finden zumeist in dieser Phase Selbstbeurteilungsverfahren Anwendung. Da bei der Identifizierung von Risiko- personen vor allem auch dem Hausarzt/Allgemeinmediziner eine wichtige Funktion zukommt, ist das Primary Care Evaluation of Mental Disorders (PRIME-MD) von Spitzer et al. ([5]; dt. Version von Pfizer, Karlsruhe) zu nennen. Es besteht aus einem Patientenfragebogen und einem Diagnostikerleitfaden für den behandelnden Arzt für die 5 wichtigsten Störungsgruppen nach DSM-IV. Unter anderem existiert ein Modul „Alkoholprobleme“ bestehend aus 4 Screeningfragen (u.a. „... habe ich gedacht, dass ich weniger Alkohol trinken sollte.“), die bei Bejahung dann durch den Arzt nachexploriert werden müssen (Dauer ca. 5-10 Minuten) und bei Bestätigung die vorläufige Diagnose „vermuteter Alkoholmissbrauch/Alkoholabhängigkeit“" gestellt werden kann. Verschiedene Studien (u.a. [5-8]) unterstreichen die Brauchbarkeit des PRIME-MD als ein Screeninginstrument. Auf weitere $\mathrm{zu}$ Screeningzwecken einsetzbare Verfahren sei auf die Überblicksarbeit von Schwoon \& Krausz [9] verwiesen.

Selbstbeurteilungsverfahren können jedoch, wie bei anderen Störungen auch, alleine nicht zur Diagnosenstellung eingesetzt werden. Ihre Anwendung muss sich einer differenzierten Nachuntersuchung anschließen, wozu sich die nachfolgend genannten Instrumente besser eignen. Hierbei soll nur auf diejenigen Verfahren Bezug genommen werden, für die es auch publizierte deutschsprachige Versionen gibt.

\section{Checklisten}

Als Erstes wurde von Dittmann et al. [10] die sog. ICD10-Merkmalsliste (ICD ML) vorgelegt. In einer separaten Sektion „Störungen durch psychotrope Substanzen“ werden die relevanten Kriterien aufgelistet, die es in einem klinischen Interview zu erfragen gilt. Die Liste wurde in einer Multicenterstudie erprobt (zu den Ergebnissen für den Bereich der Störungen durch psychotrope Substanzen vgl. [11]).

Die elaboriertesten Listen sowohl zur ICD-10 als auch DSM-IV wurden von Hiller et al. [12,13] mit den sog. Internationalen Diagnosen Checklisten (ICDCL) für ICD-10 bzw. DSM-IV vorgelegt. Für ICD-10 lassen sich z.B. folgende Störungen erfassen: Alkoholabhängigkeit, Abhängigkeit von Drogen/Medikamenten, akute Intoxikation, Entzugssyndrom, Delir sowie psychotische Störung durch psychotrope Substanzen. Reliabilitätsstudien weisen auf gute Kennwerte zur Interrater-Relia-

Tab. 1 Diagnostische Instrumente zur Klassifikation von Störungen durch psychotrope Substanzen

\begin{tabular}{|c|c|c|}
\hline Instrument (Abkürzung) & Autor(en) & Kennzeichen \\
\hline ICD-10-Merkmalsliste (ICDML) & Dittmann et al. [9] & $\mathrm{CL}$ \\
\hline Internationale Diagnosen Checklisten (IDCL) für ICD-10 bzw. DSM-IV & $\begin{array}{l}\text { Hiller et al. [12] } \\
\text { Hiller et al. [13] }\end{array}$ & $\mathrm{CL}$ \\
\hline ICD-10 Symptom Checkliste & Janca et al. [14] & $\mathrm{CL}$ \\
\hline Schedules for Clinical Assessment in Neuropsychiatry (SCAN) & van Gülick-Bailer et al. [15] & Strulnt \\
\hline Strukturiertes Klinisches Interview für .... (SKID) & Wittchen et al. [25] & Strulnt \\
\hline Diagnostisches Interview bei psychischen Störungen (DIPS) & Margraf et al. [18] & Strulnt \\
\hline Mini-DIPS & Margraf [19] & Strulnt \\
\hline Composite International Diagnostic Interview (CIDI) & Wittchen \& Semmler [20] & Stanlnt \\
\hline CIDI Substance Abuse Modul (CIDI-SAM) & $\begin{array}{l}\text { Cottler \& Keating ([23]; zit. nach } \\
\text { Lachner \& Wittchen [3] }\end{array}$ & Stanlnt \\
\hline DIA-X-Interviews (DIA-X) & Wittchen \& Pfister [25] & Stanlnt \\
\hline
\end{tabular}

CL: Checkliste, Strulnt: strukturiertes Interview, StanInt: standardisiertes Interview 
bilität hin. Ergänzend für die ICD-10 liegt eine so genannte ICD-10 Symptom-Checkliste für psychische Störungen (ICD-10 $S C L)$ als Klinikverfahren vor ([12]; dt. Teil der ICD-10 Checklisten für ICD-10; [14]), das eine vorläufige diagnostische Zuordnung erlaubt, wozu auch die psychotropen Substanzen gehören (Modul F01/F1: organische und symptomatische psychische Syndrome und durch psychotrope Substanzen verursachte Syndrome). Diese Liste kann der eigentlichen diagnostischen Erhebung z.B. mittels einer Checkliste vorgeschaltet werden. Der modulare Aufbau der Liste insgesamt gestattet eine zeitökonomische Verwendung, wenn eine Hypothese über das Vorliegen einer bestimmten Störung gebildet ist.

\section{Strukturierte Interviews}

Strukturierte Interviews liegen sowohl für ICD-10 als auch für DSM-III-R/DSM-IV vor. Bei den Schedules for Clinical Assessment in Neuropsychiatry (SCAN) handelt es sich um eines der offiziellen, von der WHO entwickelten umfassenden strukturierten psychiatrischen Interviews (dt. Version [15]), die auf der Present State Examination (PSE-9) basieren. Das System hat einen modularen Aufbau und deckt die meisten diagnostischen Kategorien nach ICD-10 und DSM-III-R ab. In den Sektionen 11 und 12 (vgl. auch Tab. 2), die fast identisch aufgebaut sind, werden neben den Konsumgewohnheiten (Häufigkeit, Menge und Art des Konsums) die für alle Substanzengruppen nahezu identischen Kriterien für Abhängigkeit, schädlichen Gebrauch, Intoxikations- und Entzugssyndrome erfasst. Von daher lassen sich die Störungen des Abschnitts F1 der ICD-10 problemlos anhand der Sektionen 11 für Alkohol sowie 12 für die anderen psychotropen Substanzen erfassen. Sowohl in der Sektion 11 als auch 12 dient die erste Frage als eine Art Screeningfrage, die dazu eingerichtet ist, die Sektion verlassen zu können, wenn kein Hinweis beispielsweise auf Alkoholkonsum erkennbar ist. Dies gewährleistet eine Ökonomisierung der Durchführung. Wird dieser Cut-off-Punkt jedoch übersprungen, schließt sich eine differenzierte Erfassung aller diagnostisch relevanten Kriterien an. Neben dem Interview selbst besteht das SCAN u.a. aus einem umfassenden Glossar mit Definitionen und Kommentaren, die die Arbeit mit dem Verfahren erleichtern. Umfangreiche Reliabilitätstudien stehen bisher aus (Ausnahme vgl. [16] zum Modul zu psychotropen Substanzen mit befriedigenden bis guten Ergebnissen).

Das Strukturierte Klinische Interview für DSM-IV (SKID) stellt das weltweit mit am meisten eingesetzte Instrument zur Diagnostik psychischer Störungen in Praxis wie Forschung dar (dt. Version [17]). Es besteht aus einer Version für Forscher und einer Version für Kliniker. Letztere kann zur Bestätigung der klinischen Eingangsdiagnostik, der Routinediagnostik und Dokumentation sowie zum Training Anwendung finden. Die Durchführungszeit des gesamten Interviews liegt bei ca. 100 Minuten bei stationär behandelten Patienten, bei ambulant behandelten Patienten etwas niedriger bei ca. 74 Minuten, bei Verwendung einzelner Sektionen verkürzt sich die Durchführungszeit entsprechend, wie beispielsweise bei der Sektion E „Abhängigkeit von psychotropen Substanzen“. Auch hier ermöglichen Screeningfragen zu Beginn der Sektion ein Überspringen, wenn kein Verdacht auf auffälligen Alkohol- oder Drogen- oder Medikamentenkonsum besteht.
Das Diagnostische Interview für Psychische Störungen (DIPS) von Margraf et al. [18] stellt eine Kombination von klassifikatorischer (kategorialer) Diagnostik sowie der Erhebung therapierelevanter Informationen, vor allem für den Angstbereich, dar, wobei sich das Spektrum erfassbarer Störungen auf den psychotherapeutischen Bereich zentriert. Das DIPS erlaubt jedoch auch ein Screening für Alkoholismus und Drogenmissbrauch. Für das DIPS liegt zudem eine Kurzversion vor, das Mini-DIPS [19]. Es hat die Funktion einer raschen, überblicksartigen Erfassung der für den psychotherapeutischen Bereich wichtigsten psychischen Störungen nach DSM-IV und ICD-10. Bei einer geringen Dauer von ca. 30 Minuten erlaubt es dennoch eine hinreichend reliable Erfassung von Diagnosen (u.a. Substanzabusus). So ergibt sich z.B. für „Substanzmissbrauch/Abhängigkeit" ein Kappa (Übereinstimmung mit klinischen Diagnosen) von 0,89, was als hoch zu bewerten ist.

\section{Standardisierte Interviews}

Auch das Composite International Diagnostic Interview (CIDI) als standardisiertes Interview wurde wie das SCAN in enger Kooperation mit der WHO und deren Arbeitsgruppen entwickelt (dt. Version [20]). Das CIDI wurde zunächst primär für epidemiologische Studien entwickelt. Inhalt wie Struktur des Interviews lassen das Instrument als ein hochgradig standardisiertes Instrument erscheinen. Der Gebrauch des Interviews sollte durch erfahrene Trainer erfolgen und kann, wenn das Training entsprechend umfangreich ist, auch durch trainierte Laien erfolgen. Die Dauer der Erfassung rangiert zwischen ein und drei Stunden. Eine computerisierte Version erleichtert zusätzlich die Anwendung. Die Anwendbarkeit sowie die Reliabilität des Instruments wurde in einer Vielzahl von Feldstudien geprüft. Hinreichende bis sehr gute Reliabilitätskennwerte (Kappa größer 0,60) wurden meist für die Sektion für psychotrope Substanzen erreicht (vgl. allgemein auch [21]). Seit seiner Entwicklung hat das CIDI verschiedene Weiterentwicklungen erfahren, von denen einige kurz genannt sein sollen. Von Kessler et al. [22] wurde eine CIDI-Short Form (CIDI-SF) publiziert. Mittels mathematischstatistischer Verfahren wurden sog. „stem questions“ für 8

Tab. 2 Sektionen zur Beurteilung von Störungen durch psychotrope Substanzen in strukturierten und standardisierten Interviews

Verfahren* Sektionen

$\begin{array}{ll}\text { SCAN } & \text { Sektion } 11 \text { „Alkoholkonsum“ } \\ & \text { Sektion } 12 \text { "Sonstiger Drogenkonsum (außer Alkohol)“ } \\ \text { SKID } & \text { Sektion E „Abhängigkeit von psychotropen Substanzen“ } \\ \text { DIPS } & \text { Screening für Alkoholismus und Drogenmissbrauch; } \\ & \text { Tabak-/Koffein-Konsum und Medikamentengebrauch } \\ \text { Mini-DIPS } & \text { "Problembereich Alkohol, Medikamente und Drogen“ } \\ \text { CIDI } & \text { Sektion B „Störungen durch Tabak“" } \\ & \text { Sektion I "Störungen durch Alkohol“ } \\ & \text { Sektion L „Störungen durch andere psychotrope } \\ & \text { Substanzen“ } \\ & \text { Sektion B „Störungen durch Tabak“ } \\ \text { DIA-X } & \text { Sektion I "Störungen durch Alkohol“ } \\ & \text { Sektion L „Störungen durch andere psychotrope } \\ & \text { Substanzen“ }\end{array}$

* Abkürzungen vgl. Tab. 1 
Tab. 3 Reliabilitätsangaben zum CIDI/CIDI-SAM und DIA-X im Hinblick auf Störungen im Zusammenhang mit psychotropen Substanzen (Beispiele)

\begin{tabular}{lll}
\hline Instrument* & Autor(en) & Ergebnisse \\
\hline CIDI & Wittchen [21] & $\begin{array}{l}\text { Test-Retest-Reliabilität für DSM-III-R } \\
\text { Kappa }>0,73\end{array}$ \\
CIDI-SAM & Cottler [23] & $\begin{array}{l}\text { Test-Retest-Reliabilität für DSM-III-R } \\
\text { mittlere Kappa 0,82 }\end{array}$ \\
DIA-X & Wittchen \& & $\begin{array}{l}\text { Test-Retest-Reliabilität für ICD-10 } \\
\text { Kappa 0,65 (Abhängigkeit von Dro- } \\
\text { gen) bis 0,78 (Nikotinabhängigkeit) }\end{array}$
\end{tabular}

* Abkürzungen vgl. Tab. 1

DSM-IV-Störungen selegiert (u.a. für Alkohol und Drogenabhängigkeit) mit dem Ziel eines ökonomischen Screenings (10 Minuten im Vergleich $\mathrm{zu}$ mindestens 1 Stunde des gesamten CIDI). Wenngleich Sensitivität (>77) und Spezifität (>96) hoch sind, stellt das CIDI-SF keinen Ersatz für umfassendere Instrumente dar, ist jedoch als Screening in epidemiologischen Studien als zeitökonomisches Verfahren anzusehen.

Im Hinblick auf die Erfassung von Störungen durch psychotrope Substanzen ist das CIDI-SAM (Substance Abuse Modul) noch zu erwähnen (vgl. im Detail [3]). Es enthält substanzspezifische Filterfragen zu Häufigkeit und Menge des Konsums von Alkohol, Medikamenten, Drogen und Tabak. Außerdem werden Beginn, Entwicklung und gegenwärtiger Konsum sowie somatische, psychische und soziale Folgen dokumentiert. Die Durchführungsdauer liegt zwischen 10 und 90 Minuten [23], die Auswertung kann per Computer erfolgen. Die Interrater-Reliabilität sowohl für die diagnostische Globaleinordnung als auch für die Symptomfragen waren hoch $(0,93$ und 0,94$)$, auch die Retest-Reliabilität ist zufrieden stellend (0,73 bzw. 0,79). Die Übereinstimmung (Kappa) mit klinischen Diagnosen betrug 0,81 für Alkohol und 0,91 für Opiate. Erwartungsgemäß ergaben sich für die Bereiche Missbrauch/schädlicher Gebrauch geringere Übereinstimmungen $[24,3]$.

Die gegenwärtig elaborierteste Version und Weiterentwicklung des CIDI stellt das so genannte DIA-X von Wittchen \& Pfister [25] für Diagnosen nach ICD-10 und DSM-IV dar. Es handelt sich dabei um ein komplexes System, bestehend aus verschiedenen Modulen wie Screening-Fragebogen sowie standardisierten Interviews, die in Paper-Pencil-Version respektive in computerisierter Version vorgelegt werden können. Das DIA-X Interview für den PC besteht aus mehreren Modulen, die neben dem eigentlichen Interview das Diagnostikprogramm, ein Programm für den Ausdruck des kompletten Interviews (inkl. Fehlerprüfung) und eine Datenbank für die weitere Verwendung der erhobenen Variablen enthalten. Für Paper-Pencil- wie Computerversion existieren entsprechende Sektionen zur Erfassung von psychotropen Substanzen. Ausgegeben werden Diagnosen nach DSM-IV mit den entsprechenden ICD-10-Codes. Reliabilitätsangaben belegen auch hier eine hohe Interrater-Reliabilität, was als entscheidendes Kriterium in der Evaluation diagnostischer Entscheidungsprozesse anzusehen ist. In Tab. 3 finden sich einige Reliabilitätsangaben sowohl für DIA-X als auch die anderen
Versionen des CIDI. Diese unterstreichen konsistent, dass für den Bereich der Störungen durch psychotrope Substanzen mittels standardisierter Verfahren zuverlässige diagnostische Zuordnungen möglich sind.

\section{Schlussbemerkung}

Mit der Einführung vor allem strukturierter und standardisierter diagnostischer Interviews zur klassifikatorischen Diagnostik und der damit verbundenen Verbesserung der Interrater-Reliabilität ist es in zunehmendem Maße möglich, klassische Fehlerquellen der psychopathologischen Diagnostik und Klassifikation, wie Informations- und Beobachtungsvarianz, zu reduzieren. Die Anwendung von Interviews setzt jedoch ein umfassendes, meist mehrtägiges Training voraus, die Anwendung strukturierter Interviews zudem klinische Erfahrungen. Unter Anwendungsgesichtspunkten weist Jung [26] darauf hin, den Zeitpunkt des Interviews zu bedenken. Werden z.B. Interviews während der ersten Woche nach der stationären Aufnahme durchgeführt, so birgt dies die Gefahr in sich, dass Symptome, die sich aufgrund des akuten Entzuges einstellen, als Zeichen für eine vorliegende Angst oder affektive Störung interpretiert werden. Ergebnisse legen nahe, dass es extrem schwierig ist, nach kurzer Abstinenzdauer reliable psychiatrische Diagnosen bei Substanzabhängigen zu stellen. Zudem sind gerade bei diesen Störungsgruppen Aspekte wie allgemeine Urteilsfehler (z.B. soziale Erwünschtheit) wie spezielle Motive (z.B. Dissimulation/Bagatellisierung) zu bedenken (vgl. hierzu auch [26]), diese lassen sich jedoch auch mittels Interviewverfahren kaum kontrollieren.

Zusammenfassend bleibt festzuhalten, dass durch die Einführung operationaler Diagnosen in etablierten Diagnosesystemen, wie ICD-10 und DSM-IV, eine zuverlässige Erfassung möglich ist. Eine weitere Reliabilitätssteigerung der diagnostischen Zuordnung ist jedoch nur durch die Anwendung von Instrumenten gewährleistet, wobei hierzu vor allem die strukturierten und standardisierten Interviews gehören. Die meist modular aufgebauten Instrumente erlauben bei Bedarf eine zeitökonomische Erfassung der interessierenden Störungen, bergen bei Anwendung einzelner Module jedoch dann die Gefahr, komorbide Störungen zu übersehen. Im Hinblick auf eine umfassende diagnostische Evaluation der Störung der psychotropen Substanzen ist jedoch weiterhin die Anwendung von zusätzlichen Instrumenten notwendig (vgl. [9]). Erst diese gewährleisten, dass die verschiedenen Facetten der Störung umfassend abgebildet werden können.

\section{Literatur}

${ }^{1}$ Freyberger HJ, Stieglitz RD. Diagnostic instruments for the assessment of disorders due to a psychoactive substance. European Addiction Research 1996; 2: 124-128

2 Üstün TB, Wittchen HU. Instruments for the assessment of substance abuse disorders. Current Opinion in Psychiatry 1992; 5: $412-419$

${ }^{3}$ Lachner G, Wittchen HU. Das Composite International Diagnostic Interview Substance Abuse Modul 2 (CIDI-SAM). Ein neues Instrument zur klinischen Forschung und Diagnostik. In: Mann K, Buchkremer G (Hrsg). Sucht, Grundlagen, Diagnostik Therapie. Ulm: Fischer, 1998: 147-156 
${ }^{4}$ Wittchen HU, Freyberger H, Stieglitz RD. Interview. In: Stieglitz $\mathrm{RD}$, Baumann U, Freyberger HJ (Hrsg). Psychodiagnostik in Klinischer Psychologie, Psychiatrie, Psychotherapie. Stuttgart: Thieme, 2001: (im Druck)

${ }^{5}$ Spitzer RL, Williams JBW, Kroenke K, Linzer M, Verloin de Gruy F, Hahn SR, Brody D, Johnson JG. Utility of a new procedure for diagnosing mental disorders in primary care. The PRIME-MD 1000 study. JAMA 1994; 272: 1749-1756

${ }^{6}$ Spitzer RL, Kroenke K, Williams JBW and the Patient Health Questionnaire Primary Care Study Group. Validation and utility of a self-report version of PRIME-MD. The PHQ Primary Care Study. JAMA 1999; 282: 1737-1744

${ }^{7}$ Stieglitz RD, Freyberger HJ. Selbstbeurteilungsverfahren. In: Stieglitz RD, Baumann U, Freyberger HJ (Hrsg). Psychodiagnostik in Klinischer Psychologie, Psychiatrie, Psychotherapie. Stuttgart: Thieme, 2001: (im Druck)

${ }^{8}$ Loerch B, Szegedi A, Kohnen R, Benkert O. The Primary Care Evaluation of Mental Disorders (PRIME-MD), German Version: A comparison with the CIDI. Journal of Psychiatric Research 2000; 34: $211-220$

${ }^{9}$ Schwoon DR, Krausz M. Diagnostik von Störung durch psychotrope Substanzen. In: Stieglitz RD., Baumann U, Freyberger HJ (Hrsg). Psychodiagnostik in Klinischer Psychologie, Psychiatrie, Psychotherapie. Stuttgart: Thieme, 2001: (im Druck)

${ }^{10}$ Dittmann V, Freyberger HJ, Stieglitz RD, Zaudig M. ICD-10-Merkmalsliste. In: Dittmann V, Dilling $\mathrm{H}$, Freyberger $\mathrm{HJ}$ (Hrsg). Psychiatrische Diagnostik nach ICD-10 - klinische Erfahrungen bei der Anwendung. Bern: Huber, 1992: 185-216

${ }^{11}$ Trabert W, Dittmann V, Mombour W. Störungen durch psychotrope Substanzen (Abschnitt F1). In: Dittmann V, Dilling H, Freyberger HJ (Hrsg). Psychiatrische Diagnostik nach ICD-10 klinische Erfahrungen bei der Anwendung. Bern: Huber, 1992: 45-68

${ }^{12}$ Hiller W, Zaudig M, Mombour W. ICD-10 Checkisten (IDCL für ICD-10). Bern: Huber, 1995

${ }^{13}$ Hiller W, Zaudig M, Mombour W. Internationale Diagnosen Checklisten (IDCL) für DSM-IV (IDCL für DSM-IV), 1997

14 Janca A, Üstün TB, van Drimmelen J, Dittmann V, Isaac M. The ICD-10 symptom checklist for mental disorders (Version 2.0). Geneva: WHO, 1994

${ }^{15}$ Gülick-Bailer Mv, Maurer K, Häfner H. Schedules for Clinical Assessment in Neuropsychiatry (SCAN). Bern: Huber, 1995

${ }^{16}$ Easton C, Meza E, Mager D, Ulüg B, Kilic C, Gögus A, Babor T. Test-retest reliability of the alcohol and drug use disorder sections of the Schedules for Clinical Assessment in Neuropsychiatry (SCAN). Drug Alcohol Dependence 1997; 46: 187-194

17 Wittchen HU, Zaudig M, Fydrich T. Strukturiertes Klinisches Interview für DSM-IV. Achse I und II. Handanweisung. Göttingen: Hogrefe, 1997

${ }^{18}$ Margraf J, Schneider S, Ehlers A. Diagnostisches Interview bei psychischen Störungen (DIPS). 2. Auflage. Berlin: Springer, 1994

${ }^{19}$ Margraf J. Mini-DIPS. Diagnostisches Kurzinterview bei psychischen Störungen. Berlin: Springer, 1994

${ }^{20}$ Wittchen HU, Semmler G. Composite International Diagnostic Interview (CIDI). Weinheim: Beltz, 1997

${ }^{21}$ Wittchen HU. Reliability and validity studies of the WHO Composite International Diagnostic Interview (CIDI). A critical review. Journal of Psychiatric Research 1994; 28: 57-84

${ }^{22}$ Kessler R, Andrews G, Mroczek D, Üstün B, Wittchen HU. The World Health Organisation Composite International Diagnostic Interview Short-Form (CIDI-SF). International Journal of Methods in Psychiatric Research 1998; 7: 171-185

${ }^{23}$ Cottler L. The CIDI and CIDI Substance Abuse Module (SAM): cross-cultural instruments for assessing DSM-III, DSM-III-R and ICD-10 criteria. Research Monographs 1991; 105: 220-226
${ }^{24}$ Cottler L. Comparing DSM-III-R and ICD-10 substance abuse disorders. Addiction 1993; 88: 689-696

${ }^{25}$ Wittchen HU, Pfister E. DIA-X-Interviews: Manual für Screening-Verfahren und Interview: (a) Interview Längsschnittuntersuchung (DIA-X-Lifetime); (b) Ergänzungsheft (DIA-X-Lifetime); (c) Interviewheft Querschnittuntersuchung (DIA-X-12 Monate); (d) Ergänzungsheft (DIA-X-12 Monate); (e) PC-Programm zur Durchführung des Interviews (Längs- und Querschnittsuntersuchung); (f) Auswertungsprogramm. Frankfurt: Swets Testservice, 1997

${ }^{26}$ Jung M. Alkoholabhängigkeit und psychiatrische Co-Morbidität. In: Mann K, Buchkremer G (Hrsg). Sucht. Grundlagen - Diagnostik - Therapie. Ulm: Fischer, 1998: 157-167

Prof. Dr. rer. nat. Rolf-Dieter Stieglitz

Kantonsspital

Psychiatrische Universitätspoliklinik

Petersgraben 4

4031 Basel

Schweiz

E-mail: rstieglitz@uhbs.ch 